\title{
Urgences
}

\section{Un t d'trop}

\section{Jacques Daignault}

Numéro 23, avril 1989

Lisière du livre

URI : https://id.erudit.org/iderudit/025520ar

DOI : https://doi.org/10.7202/025520ar

Aller au sommaire du numéro

Éditeur(s)

Urgences

ISSN

0226-9554 (imprimé)

1927-3924 (numérique)

Découvrir la revue

\section{Citer cet article}

Daignault, J. (1989). Un t d'trop. Urgences, (23), 89-99.

https://doi.org/10.7202/025520ar

Ce document est protégé par la loi sur le droit d'auteur. L’utilisation des services d'Érudit (y compris la reproduction) est assujettie à sa politique d'utilisation que vous pouvez consulter en ligne.

https://apropos.erudit.org/fr/usagers/politique-dutilisation/
Cet article est diffusé et préservé par Érudit.

Érudit est un consortium interuniversitaire sans but lucratif composé de l’Université de Montréal, l'Université Laval et l'Université du Québec à Montréal. Il a pour mission la promotion et la valorisation de la recherche. https://www.erudit.org/fr/ 


\section{JACQUES DAIGNAULT \\ - Un t d'trop}

Plan

Protée de l'alphabet, lettre épicène

$t$ voyageur comme si et pour vrai

shifteur grammatical ressuscitant les mythes

foncteur nu du sens

échangeur de lui-même

Prométhée, comme un livre

et son tirage

détaché du marché

et tous mes «t» dans une cave

chiasme saussurien

sur fond découvert

et profit bancal

abhumanisme intégral

baudrier mis à l'écart

"ab la soie et la mie et l'a»"

qui riait du coup de $D$

par avance, comme un destin

ME-NING du t

et restes inchoatifs

mais oblitérés, impostables

Pivot des lettres sans majuscules

pagure ou licorne phallique

le «t» débande en février 85

Et la pythie, rousse, se tait

Avec elle au bord de l'eau

Épiméthée chante le nouveau solfège

la boîte est pleine de $t$

\section{De l'auteur...}

Je suis embarrassé. On en sera vite convaincu. Des mois de travail; je ne compte plus les heures. Et des années derrière; encore autant devant, peut-être. Impossible de compter les heures. Le temps n'a plus de sens. La mémoire est en panne. 
Aspirée dans une fissure: infarctus de l'âme. Fêlure troublante, aussi. Bientôt le chaos. Encore un tour et le muscle, à gauche, ne tiendra pas; ou les nerfs. Ou les deux. L'ordre n'a plus de sens; ne peut plus en avoir. Mais peut-être a-t-il de l'importance. Le désordre est au comble. Mais il n'est pas sûr que je m'enfonce; le fond, plutôt, monte en surface.

Et d'où vient ce fond? De quel abîme est-il la profondeur, ou même simplement l'étendue? Combien de niveaux, de métastases dans ces décrochages persistants? Le vide est-il au bout? Y suis-je déjà? Cancer de l'âme. Le vide serait-il plein de cet embarras-là? Ou le plein ne serait-il que le vide d'ordre? Quelque chose a dû arriver.

D'où vient ce désordre? Un tiret fera l'affaire: un titre qui se prend pour une hypothèse. Trop de jeux de mots: lus, écrits. Saussure, on le sait, n'a pas tenu le coup. On dit que son hypothèse n'était pas bonne: surchargée d'intentions. Que d'hésitations sur un titre! On dit que les probabilités auraient eu raison d'elle. Mais Saussure? ou l'hypothèse? Pas maintenant. J'écris sous la dictée de ses lectures. J'ai risqué les anagrammes, les homophones, les mots-valises, les restes - par soustraction d'hétérogrammes -, les étymologies - les vraies, les fausses et toujours par dictionnaires interposés -, les acrostiches; j'ai pratiqué ces machineries du mélange - et dans plusieurs langues, les traitant comme on peut - , cette alchimie des signes, cette grammatologie appliquée que Saussure croyait à l'oeuvre, en secret, dans la poésie ancienne. Cette alchimie des grammes qu'il laissera tomber, finalement, plus inquiet que déçu: à cause du silence des réponses à ses réponses, la confirmation. On imagine Saussure stoïcien: les réponses d'un côté et les questions de l'autre; comme avec les causes et les effets ${ }^{3}$.

J'écris sous la dictée de Saussure. Et comme Roussel, à l'envers de Saussure. J'essaie peut-être de sauver la différence elle-même, le passage lui-même des Anagrammes à la Sémiotique; Roussel avant de mourir.

Roussel avait une méthode, un procédé d'écriture ${ }^{4}$. On ne sait plus compter ceux et celles qui en ont, qui en avaient: d'analogues et de différents. Saussure traduit Roussel, avant lui: Serres pourrait l'expliquer'; à l'envers. Foucault le savait ${ }^{6}$. Je traduis le traducteur. J'écris peut-être sous sa dictée. Question de rythme. Cela doit s'entendre pour qui l'a un peu lu. J'écris sous la dictée d'une âme soûle, trois fois, d'écriture. Je lis Rousseau. Un reste. 
Autre alchimie des signes. Roussel-Rousseau-Saussure $=\ll \mathrm{a}$ soul», saoul.

Trois cents pages de cette machinerie-là. Et pour la plupart illisibles; aurais-je même un jour la patience de les lire? Le Sud produit des anagrammes parfaites: les lettres sont consumées au fur et à mesure de leur production. La chaleur devient froide quand on ne sait plus si la neige est brûlante: à force d'habiter l'espace clos des murs, en plein été. On confond les pôles avec les langues étrangères; la sienne qui ne s'entend même plus. Sauf en dedans. Mais un intérieur ébranlé, menacé par tant de passages et d'habitudes rompues. Une âme soûle, trois fois, d'écriture. Diffuse. Deleuze ${ }^{7}$, sous la dictée de Foucault, pourrait l'expliquer. Je traduis l'interprète: le divers est pur dehors, sans rapport à aucun dedans; je suis l'effet d'un pli et d'une suture qui ne résistera plus longtemps aux forces du passage. Je suis à bout de souffle. L'intériorité se disous. Je suis perdu. Confus. Alors j'écris. Impossible de ponter l'âme; l'infarctus est sévère. J'oublie jusqu'à mon nom. Une vague, dépliée, emporte les lettres. Un coup de dés. Jactus linguae ad $Q^{8}$. Un coup de dés du langage; $10^{18}$ fois la chute des lettres. J'avais raison; impossible de compter les mots jusque là. La signature est la même; mais je me suis oublié, à son bord. Ne restent donc que les lettres du bord: $\mathrm{D}$ et t. Entre les deux, la brebis s'égare dans l'orthographe incorrecte. Du D, un dictionnaire: commencé déjà; à poursuivre indéfiniment. Reste donc le t. En voici l'histoire abrégée; une traduction acceptable.

\section{... en passant par l'ouvrage, ...}

Février 1985. Pour une esthétique de la pédagogie ${ }^{9}$ paraît enfin. L'imprimé me renforce l'âme. Mille fois mon nom en page de couverture. La suture est solide, on y croirait Narcisse. L'imprimé provoque l'expression publique d'un «Je». Un livre comme preuve d'existence; quelques pages, au moins, qui seraient venues à bout de la confusion: une reconnaissance publique. Enfin la diffusion. L'âme heureuse de se reconnaître. J'étais celle-là. Pour une heure ou deux. Seulement. Une force allait encore une fois déplier ma certitude: l'erreur, je veux dire l'errance typographique. Après sept années d'effort à comprendre l'expression «médiation de pertinence", un «t» d'trop, en page couverture quatre - au recto de ma signature - transformait la médiation en méditation. On ne pouvait pas si bien dire. Et pour le pire; du moins l'ai-je d'abord cru. J'avais beau me répéter que le concept était sauvé à l'intérieur du livre, l'exhibition de l'erreur, au recto de ma signature, s'imposait comme symbole d'un échec. Et je scrutais le livre à la loupe. J'avais 
pourtant payé le gros prix pour une lecture d'épreuve sans faille. J'avais dû mal comprendre ce que cela signifiait; une lecture à la lettre ne pouvait que distinguer les mots des non-mots: un mot bien orthographié aux plans lexical et grammatical et le même mot mal orthographié. Méditation n'était qu'un autre mot. Et bien orthographié. Personne n'était à blâmer. L'anagramme de mon nom - il m'aura fallu trois ans pour le comprendre - le dit sans détour: un coup de dés du langage. Et s'il faut en croire le nombre $\left(10^{18}\right)$, je ne suis pas au bout de mes peines. En effet. L'âme heureuse allait devoir fonder son bonheur sur autre chose que la suture de Narcisse. Des erreurs, il y en avait d'autres. Et je ne compte plus les genres, ni les espèces: de la lettre à l'éditeur, en passant par la thèse elle-même. Je dois être né pour l'embarras. J'écris sous la dictée de mes erreurs.

L'éditeur m'a bien eu. Mille copies devaient coûter dix mille dollars. J'en ai payé la moitié: cinq mille. L'éditeur, à mon insu, en payait trois mille seulement; il me laissait croire néanmoins à deux mille de plus. Première erreur: illusion d'un partage équitable des coûts. II m'aura fallu deux ans pour découvrir l'hypocrisie; deux ans pendant lesquels l'éditeur ne me payait pas mes droits non plus. Mai 1988: une année encore et le conflit allait se régler par une annulation de contrat. Je dispose maintenant de près de sept cents copies à donner. L'éditeur a fait ses frais (plus un profit raisonnable) et m'a retourné les copies invendues. J'ai perdu environ trois mille dollars. On dirait une sale histoire; elle n'a pourtant rien de tragique. II ne s'agit, après tout, que d'un mauvais placement: une perte de capital. Soyons beau joueur. Et puisque tout s'échange, convenons au moins de ceci: le «t» d'trop m'a coûté peut-être davantage. II m'a sauvé plus d'une fois de la confusion absolue. Cela ne fait pas loin d'une thèse.

Le «t» d'trop n'est pas isolé. II s'écrirait sous la dictée de Foucault: un énoncé ${ }^{10}$. Quatre autres erreurs typographiques ${ }^{11}$ ont été repérées jusqu'ici. "était/étant», «en/ne», «monadisation/nomadisation» et «Lapassage/Lapassade». Elles ont en commun l'équilibre du bilan; rien de trop, rien de moins. Pur échange de lettres: par inversion ou par substitution. Échange purement matériel de lettres entre elles; sans profit, sans perte. Pur échange entre des monnaies. La monnaie sans valeur et sans usage. Monnaie périmée: le vers Saturnien; les anagrammes de Saussure. Translitération des signes. Mais cela s'échange-t-il contre quelque chose? La question est difficile. Tout le texte va se jouer sur elle. Le «t» d'trop pourra-t-il, en effet, se libérer de l'économie du capital sans retomber sur la fiction de l'usage? Baudrillard pourrait 
l'expliquer ${ }^{12}$. Roussel l'a vécu. J'écris sous la dictée des éclairs. Un flash après l'autre. Je sais l'importance des détours. II faut s'armer de patience. L'éclair est si fragile, malgré sa puissance.

J'ai dit quatre erreurs de plus: «i/n», «e/n et $n / e », ~ « m / n$ et $n / m »$, $g / d »$. Le signe «/" signifie «à la place de». Je ne l'ai pas pris au hasard. II tient aussi d'une erreur. Chez Rousseau cette fois-ci. Et dans un ouvrage peu connu: le Projet concernant de nouveaux signes pour la musique ${ }^{13}$ de 1742 . La première fois qu'il utilise ledit signe, Rousseau est grammairien de son vocabulaire. «/» vaut pour un dièse. «Le dièse s'exprime par une petite ligne qui croise la note en montant de gauche à droite. Sol dièse, par exemple, s'exprime ainsi 5 , fa dièse, ainsi 4». La deuxième fois, Rousseau est victime d'une erreur. "À la place de», ne signifie plus ce que signifie le signe en général, mais constitue simplement une "erreur». "Le bémol s'exprime ainsi par une semblable ligne qui croise la note en descendant 7,2 ». Substitution du signe par inversion. Le mauvais signe: le risque de toute translitération. L'erreur anagrammatique plutôt que sémiotique. Le passage entre les deux Saussure. Le passage est difficile. Risqué, surtout. Roussel s'est suicidé; peut-être. Saussure a tué, peut-être. Je veux dire que la raison sémiotique n'est pas innocente. La chasse aux démonstrations rigoureuses, aux confirmations de toutes sortes est à prendre à la lettre. "Chez Platon, et pour une tradition qui perdure jusqu'à l'âge classique, la connaissance est une chasse. Mettre à mort le gibier, pour le manger, au fond des bois [...] Ces épistémologies ne sont pas innocentes: elles demandent des têtes, au tribunal critique [...] Savoir, c'est mettre à mort [...] le je qui jouait à l'agneau, en minimisant ses pouvoirs, et plaçant en amont de lui les puissances dites, le je, c'est le loup même [...] L'homme occidental est un loup de science. ${ }^{14}$ Saussure juge de Ferdinand voulait des preuves de son innocence. II n'en a pas trouvé. Le poète les lui a refusées. Saussure, inquisiteur de lui-même, a condamné sans appel son hypothèse sur les anagrammes. II allait fonder la sémiotique. Le poète ne s'est pas présenté au tribunal de la critique. Et c'est heureux pour la suite du monde; la condamnation n'est encore qu'un jugement rendu sur l'accusation et pas encore sur la sentence; il ne faudrait pas répondre, cependant, à la critique du loup. Le jeu est de plus en plus serré. Le milieu est devenu l'enjeu des nouvelles épistémologies. Tout va se passer entre le suicide et le meurtre, entre une anagramme ratée et une sémiotique appliquée. À la lettre. Entre nihilisme et terrorisme. Le passage est vraiment risqué. On n'invite jamais le tiers que pour l'exclure. Et l'exclusion est d'autant plus violente que le loup s'y trouve. Je suis en danger. Lisez Le parasite $^{15}$. «Le petit chaperon rouge» ferait aussi bien l'affaire. 
Dans Pour une esthétique de la pédagogie je cherchais précisément le passage entre terrorisme et nihilisme. Ma rencontre avec un «t» d'trop a rendu le passage encore plus serré. Le coup de dé (D) de mon nom n'y est pas étranger. Entre le début et la fin de mon nom propre, entre le coup de «D» et le «t» d'trop, se trouve un mélange inquiétant «aignaul». II est facile d'y lire l'AGNeAU que le loup n'a pas encore mangé. Le Loup auquel manque le POU: le parasltE, l'«i/e» entourant le «t». Translitération qu'il est maintenant possible de transcrire. L'«i/e» répété jusqu'au «"». L'anagramme traduite dans la sémiotique. Ni l'erreur de l'une, ni l'application de l'autre, mais la traduction de l'une à l'autre: i.e. passage entre deux fois la mort évitée. Le «t» de la traduction et du tiers: inclus-exclus, plutôt inclure qu'exclure «en/ne»; comprendre la monade comme le «t» indivise du passage de "médiation» à "méditation». Médiation: inclusion du Tiers au plan du signifié, mais exclusion du «t » au plan du signifiant. Méditation: inclusion du "t» au plan du signifiant, mais exclusion du Tiers au plan du signifié. Ce que disent tous les dictionnaires.

Le «t» d'trop avait donc un sens. On lui en a trouvé un. L'âme peut panser ses blessures. Profiter d'un repos. Ça ne durera pas longtemps. L'âme est soûle, trois fois d'écriture. Saussure a son compte; qu'il repose en paix. Essayons encore quelques pas en direction du «t».

Quatre erreurs en forme de substitution; une seule en forme de supplément. Cela fait trois chaînes: le «t» tout seul; la série des inclus «ienmng» et la série des exclus «nnemnd». Le rapport entre les inclus et les exclus a donné le sens du «t». Mais il y a plus. La série des inclus est en rapport direct avec le «t»; il est possible, en effet, de le consumer: une lettre en moins pour une lettre en plus: le "a» manquant de la série des inclus. "ienmng $+a=$ MEANING. On peut même ajouter la troisième série: chapelets à la verticale: NAME et IND (index); anagramme horizontale: END. Je traduis. Le «t» de la fin est l'index du sens de mon nom.

\section{$\mathrm{N}$ \\ meaning \\ $m n$ \\ END}

La traduction est passage. Et de plusieurs manières. Je savais bien que les nouvelles sutures de l'âme ne résisteraient pas davantage que les autres; il ne s'agit jamais que de doublures. Je l'ai dit: le passage est difficile. La notion même de passage est 
diffuse. La traduction du «t» d'trop n'est pas complète; ne le sera jamais. Et pour des raisons elles-mêmes incomplètes. Combien d'erreurs, encore, dans Pour une esthétique de la pédagogie? Combien d'autres translitérations à venir au voisinage de ces erreurs? Et même combien d'autres transcriptions possibles des mêmes erreurs? Peut-être y en a-t-il de meilleures! Lisons Roussel. "Ce procédé, en somme, est parent de la rime. (...) C'est essentiellement un procédé poétique [...] Encore faut-il savoir l'employer. Et de même qu'avec des rimes on peut faire de bons ou de mauvais vers, on peut, avec ce procédé, faire de bons ou de mauvais ouvrages. " ${ }^{16}$ Roussel écrit peut-être sous la dictée de Kant: l'artiste n'a pas de méthode au sens strict, mais une manière $^{17}$. La méthode est singulière et définie: LE chemin. La manière est singulière et indéfinie: UN chemin. La traduction est toujours plurielle: CHEMINS. La différence translitérative entre "transcription» et «translitération» nous l'indique: ITER constitue le reste, par soustraction des deux mots; selon la première méthode de Saussure. «Iter», en latin, signifie le chemin («hodos» en grec), mais aussi, comme préfixe, la répétition. Je le redis autrement: $« \mid »=$ re: «t». Pour Roussel, l'anagramme était UN chemin: iter ou hodos; une manière. Pour Saussure, la sémiotique fut LE chemin, finalement: trans-iter ou meta-hodos; la méthode. Je cherche le passage dans la multiplicité des voies: iter(chemin)iter(répétition); CHEMINS (qui ne mènent nulle part?). J'essaie de transcrire les effets de la translitération. Cela fait peut-être une traduction.

«ll est possible que la science soit l'ensemble des messages optimalement invariants pour toute stratégie de traduction. Lorsque ce maximum n'est pas atteint, il s'agirait des autres aires culturelles. Systèmes déductifs, inductifs [...] demeurent les plus stables par le transport en général; sous ce seuil, les systèmes productifs, reproductifs (...) varient, chacun selon sa différence. Leur différence n'est, en fait, que la variation. ${ }^{18}$ Serres n'est pas à la recherche d'une théorie de la traduction, mais de son application dans les champs les plus divers du savoir. Toute son oeuvre le dit. II n'écrit jamais que sous la dictée des passages. "On mesure les transformations du message. Telle loi de l'histoire dit les états de la matière, tel traitement de la forme et de la couleur dit la révolution industrielle. Versions différentielles. ${ }^{19}$ Serres traducteur. Mais Serres également critique de la traduction. «Aux limites de la trahison [...] tel groupe au pouvoir parvient à détourner les messages optimalement stables, la science, pour les faire produire la mort: la thanatocratie. ${ }^{20}$ Serres critique des traductions "globales»; partisan des épistémologies "locales». Serres éthicien des passages. 
Mais de quelle définition du passage s'agit-il? Que signifie passer? Et quel rapport avec traduire? J'ai traduit le «t» d'trop. Qu'est-ce à dire? J'ai cherché le passage entre deux fois la mort évitée. Symbole, ici, d'une double réduction: à la différence (la variation) et à l'identité (l'invariant). Le marxisme a raison: le relativisme des différences peut être nihiliste; le christianisme a raison: la contradiction peut être terroriste. J'ai cherché le passage entre les deux; non pas de l'une à l'autre - et peu importe le sens - , mais leur différence elle-même (la différance aussi). J'ai traduit ma thèse à l'occasion d'un «t» d'trop.

La même thèse?

Pourquoi cette question?

Deux questions de suite; j'essaie de répondre à la seconde.

Et je ne sais qu'on me demandera pourquoi. Cela fait une troisième question:

pourquoi répondre à la question du «pourquoi» plutôt qu'à la question elle-même?

Si je pouvais répondre à la seconde, on ne poserait plus ni la première, ni la troisième. Je crains qu'on sera déçu. La traduction du «t» d'trop est une réponse à la seconde question: «pourquoi demander s'il s'agit de la même thèse?»

La traduction est passage, ici, entre des questions et des réponses au sens habituel; non pas le passage d'une question vers une réponse, mais leur différance. II n'y a donc, ici, ni question ni réponse au sens habituel; que des problèmes ${ }^{21}$. Le poète n'a pas répondu à la question de Saussure ${ }^{22}$; l'interprétation de ce silence a été un «non» clair à la question, et Saussure abandonna l'hypothèse des anagrammes. Et dans le cas de l'interprétation contraire - une réponse positive - Saussure aurait édifié une théorie. Alors que ce soit par oui ou par non, la réponse en général - témoignage d'une connaissance binaire - clôt toujours l'espace du problème. Toute réponse tue la question. J'insiste. La traduction du "t" d'trop est un problème: vivant. J'écris peut-être sous la dictée d'une vie sans organes.

L'âme ébranlée par l'erreur s'est reconstituée peu à peu; la doublure s'est refaite du côté de l'objet, de l'erreur objective. Personne n'était à blâmer. L'âme était seule face à elle-même: méditative; elle devait se constituer comme sujet dans son rapport au monde, au chaos. Dépliée par l'erreur, la marge, le hasard - la monstruosité du divers absolument - l'âme s'est repliée sur elle-même; heureuse, peut-être, d'avoir reconquis du sens. J'écris DU sens et non pas LE sens. Car, alors même qu'elle travaillait à se 
reconstituer du côté de l'objet, ça travaillait contre elle, contre son identité: le «t» d'trop n'était peut-être pas le sien complètement, mais l'héritage d'une médiation. Elle allait devoir en partager le sens.

\section{...au sujet du texte}

Février 1985. Encore une fois. L'invariant du «t» d'trop n'était pas dans le message, mais dans le médium. Dans le magazine Lire de ce mois-là, je découvre, signée Bernard Pivot, une note intitulée "Horreur! le " $t$ " avait disparu...»" Jacques Audiberti écrivait «Tu est beau». «Tu» comme sujet de la proposition et non comme sujet du verbe: le pronom «tu» est beau; par exemple. Mais par six fois les correcteurs d'épreuves ont biffé le «t»; et par six fois l'auteur a dû le rajouter. Mais c'était compter sans la persistance du «tiers". Durant l'impression, un correcteur, jetant un coup d'oeil sur une épreuve, était tombé exactement sur la phrase «Tu est beau». Alors, non seulement arrête-t-il la machine mais corrige-t-il aussi la grammaire. À cause du Tiers - encore lui -, le sujet de la proposition, un nom au sens grammatical, devenait donc irrémédiablement le sujet d'une phrase, un PROnom grammatical; l'ouvrage paraît en effet sans «t», avec un préfixe de trop: PRO. Protée de l'alphabet.

Qui oserait douter que le «t» d'trop de Pour une esthétique de la pédagogie soit celui-là même qu'ait perdu Audiberti? Une autre histoire de passage. Quelque chose a traversé la frontière, aussi large qu'on voudra, qui sépare, ici, l'Europe de l'Amérique. Le «t» ne se serait pas échangé contre un sens ou de l'argent. Aurait-il simplement changé de place? Lacan pourrait l'expliquer; symboliquement. Mais le «t», du côté de l'Europe, est d'écriture seulement; il appartient au corps du texte, la différence entre «es» et «est» est impossible à l'oreille. Et cela marque une différence. II est difficile, en effet, de reconnaître au "t» la valeur d'un échange; difficile aussi d'y voir une valeur d'usage. Que vaut quelque chose, en effet, dont l'usage est de s'échanger contre lui-même et dont l'échange n'est jamais que contre lui-même? J'écris peut-être sous la dictée d'une autre économie politique. La valeur du «t» est une valeur de passage: une note, comme en musique ${ }^{25}$, plutôt qu'un signe; la différence elle-même comme passage.

L'âme inquiétée par le «t» n'est donc plus seule dans l'embarras; elle doit partager son secret avec une autre au moins. Avec toutes peut-être. L'âme n'est plus seulement constituée par un «t», 
un texte, une trace dont la traduction serait, pour elle, le moyen par excellence de prétendre être constitutive de son sens; l'âme se prête aussi au pliage de l'autre: de l'autre âme constituée par le même "t", le même texte général dont la traduction pourrait être également constitutive de mon âme. Tout est un.

À multiplier les rapports, l'indifférencié reprend du travail. Le chaos remonte à la surface. Le fond, indifférencié, sur fond duquel les différences se constituent se présente en même temps qu'elles. Le «t» n'est pas le même que pour être la trace permanente du reste indifférencié, son archi-écriture ${ }^{26}$. Le passage ne serait rien d'autre que l'indifférencié, le chaos présenté au moins deux fois sous l'éclairage d'un flash, deux fois transcrits, sous la dictée d'une âme soûle, plusieurs fois, d'écriture. J'écris sous la dictée de Deleuze ${ }^{27}$. Le «t» n'a pas décliné son identité, il a seulement répété ses passes en surface; comme des éclairs dans un ciel noir. J'essaie de ne pas tricher avec le fond sur fond duquel se lèvent toutes ces intuitions que je transcris, ici, de mon mieux. Je ne me suis laissé embarrasser qu'à cette fin: témoigner de mon souci de rigueur et de sensualité. II n'est pas sûr qu'on va me croire. La partie est de plus en plus serrée.

Audiberti et l'abhumanisme ont gagné un lecteur; pour un temps «t» au moins.

1. J. Audiberti, «Abhumanisme», dans M. Giroud, Audiberti, coll. «Poètes d'aujourd'hui", Paris, Seghers, 1973, p. 184.

2. On comparera deux textes. J. Starobinski, Les mots sous les molts. Les anagrammes de Ferdinand de Saussure, coll. "Le chemin", Paris, Gallimard, 1971, et J. Baudrillard, L'échange symbolique et la mort, Paris, Gallimard, 1976, p. 283-309.

3. On lira E. Bréhier, La théorie des incorporels dans l'ancien stö̈cisme, Paris, Vrin, 1980. Ce texte est l'armature philosophique de Logique du sens de $\mathrm{G}$. Deleuze, coll. “Critique», Paris, Minuit, 1969.

4. Évidemment, le «Comment j'ai écrit certains de mes Livres» dans R. Roussel, Comment j'ai écrit certains de mes Livres, Paris, Pauvert, 1963, p. 9-35.

5. Je pense à la thèse principale de Serres sur la traduction et les passages.

6. M. Foucault, Raymond Roussel, Paris, Gallimard, 1963.

7. G. Deleuze, Foucault, Paris, Minuit, 1986.

8. J. Daignault, "Curriculum vitae-Biography Dictionary», conférence présentée au Department of Curriculum and Instruction, LSU, Baton-Rouge, mars 1988 (texte non publié).

9. J. Daignault, Pour une esthétique de la pédagogie, Victoriaville, NHP, 1985.

10. Et c'est bien dans cette direction de L'archéologie du savoir, Paris, Gallimard, 1969, que j'ai orienté la recherche.

11. Je ne vise que les différences entre le manuscrit remis à l'éditeur et le texte publié.

12. op.cit. II s'agit de la thèse principale de Baudrillard. L'échange symbolique - qu'on appellera plus loin la valeur de passage - est une abolition de la 
valeur et du cumul.

13. Pour apprécier la suite, il faut savoir que l'erreur dont parle mon texte se trouve sur le 7 et le 2 dans l'édition des oeuvres complètes de 1824 et sur le 2 seulement dans l'édition de 1938, qui est reproduite intégralement aujourd'hui. Je l'ai vérifié deux fois. Dans Écrits sur la musique, Paris, Stock, 1979, et dans une édition bilingue, Project concerning new symbols for music, Kilkenny (Ireland), Boethius Press, 1982. L'édition française reproduit l'erreur, et sans commentaire, alors que l'édition bilingue propose la correction en bas de page.

14. M. Serres, Hermes IV. La distribution, coll. "Critique», Paris, Minuit, 1977, p. 103-104.

15. M. Serres, Paris, Grasset, 1980.

16. R. Roussel, op. cit., p. 23.

17. E. Kant, Critique de la faculté de juger, traduction d'A. Philonenko, Paris, Vrin, 1979, p. 148 et 176.

18. M. Serres, Hermes III. La traduction, coll. "Critique», Paris, Minuit, 1974, p. 11.

19. Ibidem.

20. Ibidem.

21. "Quelle est la réponse à la question?" Le problème. Comment résoudre le problème? En déplaçant la question. Le problème échappe à la logique du tiers exclu, puisqu'il est une multiplicité dispersée». M. Foucault, «Theatrum philosophicum», Critique, Paris, novembre 1970, p. 900.

22. Saussure ne veut pas se contenter de preuves internes, il cherche une preuve externe; il croit pouvoir l'obtenir en écrivant à son collègue Pascoli de l'université de Bologne - poète italien honoré par l'académie d'Amsterdam pour ses vers en latin - dans l'oeuvre poétique duquel il trouve des anagrammes en abondance. II lui écrira deux lettres. Dans la première il ne donne aucun détail mais vise seulement à établir un contact. Dans la deuxième, il pose carrément la question: "Est-ce par hasard ou avec intention" etc. "Giovanni Pascoli laissa cette seconde lettre sans réponse: c'est du moins ce qu'assure aujourd'hui un élève ( $M$. Léopold Gautier) que Saussure avait associé à sa recherche. Le silence du poète italien ayant été interprété comme un signe de désaveu, l'enquête sur les anagrammes fut interrompue". J. Starobinski, op. cit, p. 149-151.

23. Je remercie Micheline Houde qui - quelques jours seulement après la publication de mon livre - a porté à mon attention l'existence du texte de Pivot.

24. "L.es carnets de Bernard Pivot", Lire, Paris, no 113, février 1985, p. 11.

25. La référence à la musique tient autant dans l'expression «les notes de passage" que dans les passages difficiles à jouer, ceux dont on dit que l'exécution à elle seule fait toute l'interprétation.

26. On traduira également le passage du «t» comme jeu symbolique de la présence-absence d'un supplément. Je renvois le lecteur au texte de J. Derrida, De la grammatologie, coll. "Critique», Paris, Minuit, 1967.

27. Je réfère, ici, au concept de distinction unilatérale dans G. Deleuze, Différence et répétition, Paris, PUF, 1968. 